\title{
Luces y sombras de los objetivos sanitarios para la década 2000-2010
}

Este es un tema que no puede pasar inadvertido ante la comunidad de salud pública. Por ello, en esta editorial comentamos tanto el contexto en que ha tenido lugar su implementación, como los aspectos más relevantes de la evaluación de mitad de período realizada recientemente por expertos externos.

Los Objetivos Sanitarios constituyeron el punto de partida de la reforma sectorial de salud, cuyos componentes debían articularse para el logro de metas previamente definidas. En ese contexto, en enero de 2002 el Ministerio de Salud comprometió con el entonces Presidente Ricardo Lagos, un instrumento destinado a orientar en el mediano y largo plazo las políticas, estrategias, planes y programas del sector para la década que estamos viviendo.

Igualmente, se concluyó que Chile enfrentaba cuatro desafíos sanitarios prioritarios: a) el envejecimiento progresivo de la población, con una creciente carga de patologías degenerativas de alto costo en atención médica; b) las desigualdades en la situación de salud de la población, que se traducían en una importante brecha sanitaria entre los grupos de distinto nivel socioeconómico; c) la necesidad de responder adecuadamente a las expectativas legítimas de la población con respecto al sistema de salud y d) el mantener los logros sanitarios alcanzados.

De estos desafíos se originan los cuatro grupos de Objetivos Sanitarios para la década. Una vez definidos éstos y sus metas, ha correspondido a las unidades técnicas del sector el diseño de las políticas y estrategias y su implementación, en forma de planes y programas que incluyen las garantías explicitas en salud.

La evaluación de mitad de período muestra avances, estancamientos y algunos retrocesos que vale la pena comentar, en un contexto caracterizado por la puesta en práctica de un conjunto de leyes y programas, entre los que destacan las leyes 19.937 sobre Autoridad Sanitaria y 19.966 sobre Garantías Explícitas; la implementación de los compromisos de gestión y del nuevo modelo de autoridad sanitaria; el fortalecimiento de acciones medioambientales y la disponibilidad de mayores recursos financieros.

El informe presentado por las autoridades consigna avances en la disminución de la mortalidad infantil y materna y en la reducción de la tasa de mortalidad por cáncer cérvicouterino, entre otros indicadores. Estos logros contrastan con el estancamiento en las metas relacionadas con hábitos de vida -como obesidad, tabaquismo y conducta sexual-, suicidio y accidentes de tránsito.

Asimismo, se aprecia un aumento en la brecha entre ricos y pobres en materia de salud. Por ejemplo, se indica que las mujeres sin educación tienen 26,5 veces más riesgo de morir de cáncer de mama que aquellas con más de 13 años de estudios, cifra que creció en relación al período 1998-2000. Esta inequidad se manifiesta también en que los hijos de madres con menor nivel educacional tienen tres veces mayor riesgo de morir que aquellos nacidos de mujeres con más de 13 años de enseñanza; ello, en un contexto de disminución de la mortalidad infantil que sitúa a Chile entre los países con mejores indicadores en este ámbito.

Es obvio que estos resultados se inscriben en una sociedad chilena que ha experimentado significativos cambios en los últimos años, los que deben ser reconocidos para seguir avanzando en el mejoramiento de las condiciones de vida y de salud de la población.

La evaluación de los logros sanitarios y de los factores que influyen en su evolución -sin pretender ser exhaustivos- muestra que los problemas más importantes que enfrenta el sector salud en nuestro país son la inequidad en el acceso y la calidad de las acciones de salud para diferentes 
grupos poblacionales, con diferencias de 4 a 5 veces entre diferentes servicios de salud; el incremento en la movilidad de las personas; el envejecimiento de la población, especialmente de las mujeres, y la patología que ello genera; la nueva realidad en el campo de la salud sexual y reproductiva, cuyo hecho más destacado es la iniciación de la actividad sexual a edades cada vez menores, acompañada de una baja cobertura de las acciones de anticoncepción en el hombre; y el insuficiente control de un grupo de enfermedades infecto - contagiosas emergentes, incluidas algunas entéricas.

Estos problemas generan, a su vez, un conjunto de desafíos que el sector salud debe enfrentar de manera decidida si quiere alcanzar las metas propuestas en los Objetivos Sanitarios para la década $2000-2010$.

Para ello, sugerimos priorizar: un pronto y significativo mejoramiento en la calidad y oportunidad de la atención de salud, especialmente para los grupos poblacionales más vulnerables; una mayor atención a aspectos relacionados con los recursos humanos del sector; el mejoramiento de la capacidad de respuesta frente a la amenaza de enfermedades infecciosas emergentes, especialmente mediante la dotación de laboratorios virológicos modernos; el fortalecimiento de la capacidad de respuesta de la autoridad sanitaria en el área medio ambiental y de alimentos, mediante la capacitación de personal especializado y la dotación de equipos adecuados; y la superación del desfase existente entre la gestión de los servicios y programas, la incorporación de personal idóneo en su implementación, los mejoramientos en infraestructura y la dotación de equipamiento moderno.

Finalmente, es muy importante que frente a este escenario se adopten medidas que contribuyan a asegurar la sustentabilidad de los logros sanitarios tanto desde el punto de vista técnico-científico como ciudadano. Entre otros, ello requiere establecer: i) un sistema de monitoreo, semestral o anual a nivel regional y comunal, que permita ajustar y priorizar oportunamente su avance en el tiempo y el espacio; ii) correlacionar las disposiciones de la reforma con los logros sanitarios y dar a conocer éstos a la sociedad en forma oportuna y efectiva, para aumentar su compromiso y participación y iii) establecer acuerdos del Gobierno con las instituciones formadoras de recursos humanos -universidades-, sobre los programas de formación, capacitación y actualización requeridos, el desarrollo conjunto de observatorios para el monitoreo del cumplimiento de los Objetivos Sanitarios y la realización de la investigación aplicada que permita, además de evaluar políticas y programas, generar nuevos conocimientos en este campo. 